\title{
Review of Docphin: An App for Mobile Access to Medical Journals
}

\author{
Andy Wai Kan Yeung ${ }^{1}$ (D) $\cdot$ W. Keung Leung ${ }^{1}$
}

Published online: 14 November 2016

(C) Society for Imaging Informatics in Medicine 2016

\section{App Specs}

App Icon URL: https://lh5.ggpht.com/_RTHWsWYXFacz6 whKrA-Ikcl6uUWXBdMBkwx6hVGZKvzmZpdDmGm3

T54GibaSEthRvI=w300-rw

App Name: Docphin

App Developer: Docphin

App Developer Website: https://www.docphin.com/

App Price: free

Apple App Store URL: https:/itunes.apple.com/app/docphinmedical-journals-pubmed/id542551481?mt=8

Google Play Store URL: https://play.google. com/store/apps/details?id=com.docphinapp

Category: journal

Tags: journal searching, evidence-informed practice, free, medical imaging, both iOS and Android compatible

Works Offline: N

FDA Approval: NA

Promotion Code: None

\section{Quick Review}

(1 star: lowest / 5 stars: highest)

Overall Rating (1-5): 4

Content (1-5): 5

Andy Wai Kan Yeung

ndyeung@hku.hk

1 Faculty of Dentistry, The University of Hong Kong, Hong Kong, Hong Kong
Usability (1-5): 4

Pros: The journal list is comprehensive. Individual publications can be saved for quick retrieval.

Cons: Loading time is slightly slow. It is unable to tag or comment on the abstract page of the records.

At A Glance: It comes in handy as a mobile app for accessing up-to-date literature, especially with the "Alerts" function.

\section{Full Review}

\section{Introduction}

Imaging is an evolving field with abundant technology and practice advancements, both of which are best disseminated through academic journals. Instead of browsing through the web, it will be more convenient to have a mobile app that indexes and bookmarks the favorite journals. Docphin is the app of choice, and it allows quick retrieval of relevant publications with only a few clicks on the smartphone. The Android version of the app was reviewed.

\section{Purpose/Features/Content}

The purpose of this app is to serve as a web portal that collects information from a diverse list of academic journals. At the first time of registration, the user can choose a specialty, for example, radiology. Afterwards, the app compiles the journal list accordingly. Also, after setting proxy login with the user's institution, full text articles and PDFs are readily available depending on the library subscription of the institution.

There are numerous features available from the menu (Fig. 1). It is intuitive to begin by clicking "Journals" to choose a particular journal from a user-defined list (Fig. 2) 
and browse through the table of contents from all of its issues. On the other hand, it is equally convenient by using the "Search" function in which the user can conduct a basic search with title, author, and/or journal title (Fig. 3). Advanced filters, such as article types and formats, are available for an advanced search. Shortlisted publications are labeled with a star icon and retrieved by clicking "Saved." One important feature of this app is the "Alerts." It automatically traces for new and updated publications for user-defined keyword(s) appearing in the title or author list (Fig. 4).

\section{Usability}

This app is well designed and straightforward to be used. In this case, one of the author's publications is used for the illustrations [1]. The journal NeuroImage was not on the predefined list of radiology, but it was easy to add it back from the full list of indexed journals. Both portrait (Fig. 5) and landscape (Fig. 6) modes are working well, with the default font size and line spacing suitable for prolonged reading. The abstract can be shared via numerous channels (Fig. 5). Additionally, it can be opened from the browser linking to the publisher's website, or from PubMed website.

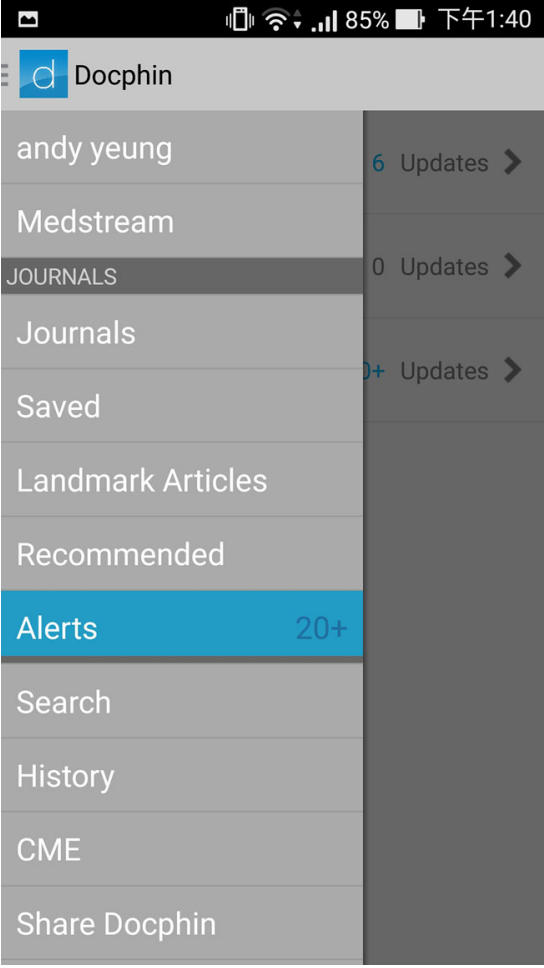

Fig. 1 Menu of the app. The full list of the menu can be displayed in a portrait mode

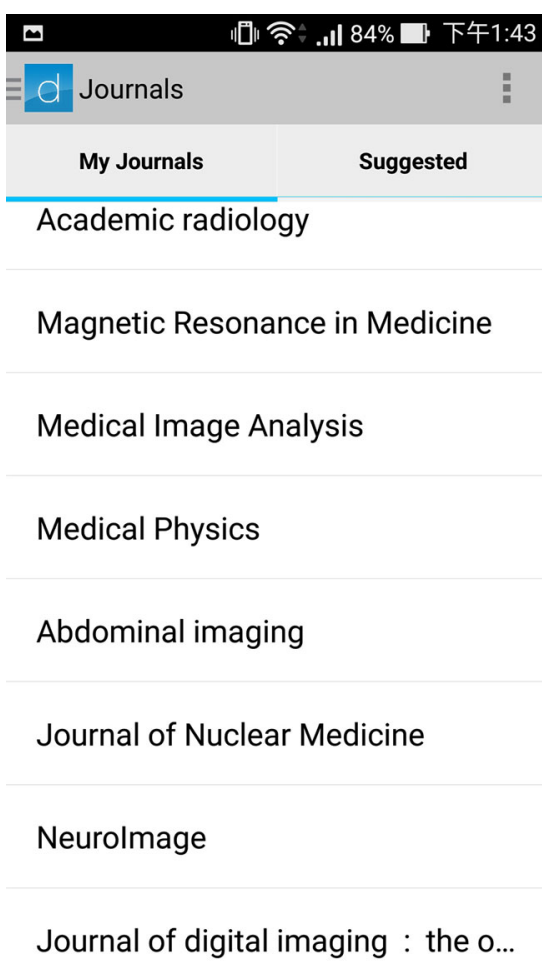

Fig. 2 Journal list. The journal list can be customized by adding or removing journals

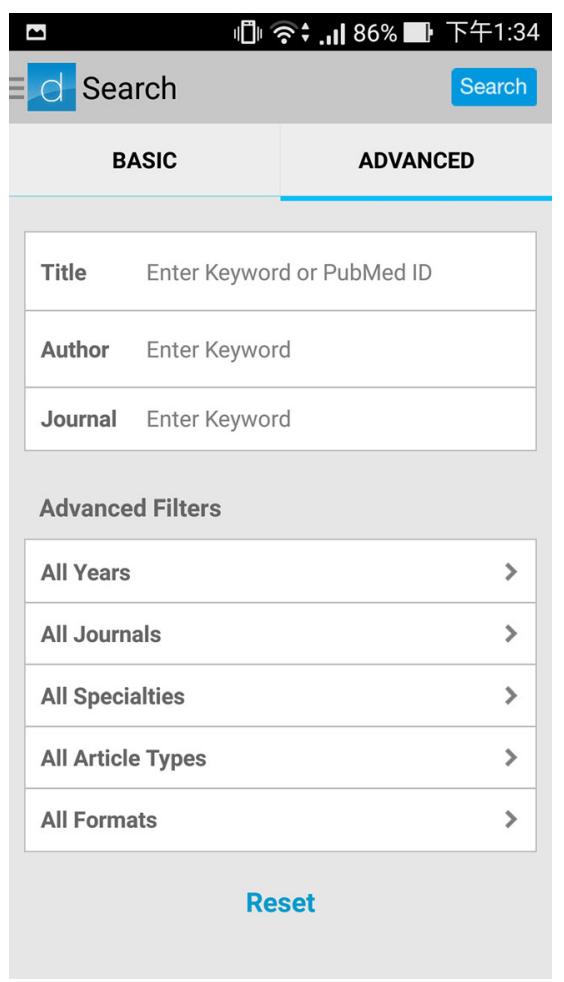

Fig. 3 Search function. There are basic and advanced search functions 


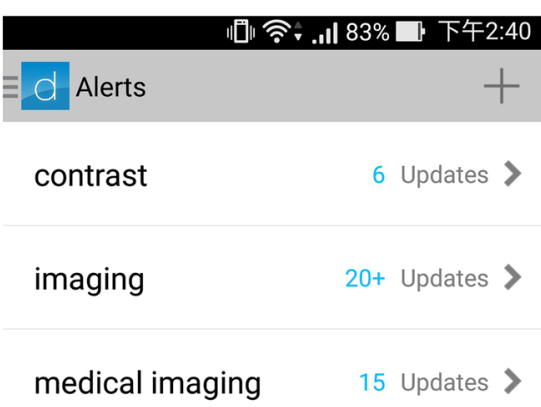

Fig. 4 Alerts function. User can define keyword(s) to be searched within title or author list, and new publications will add to the updated lists

\section{Good}

The interface is intuitive and straightforward. Every function is self-explanatory and user-friendly. The app occupies only $8 \mathrm{MB}$ of storage space, which makes it nearly possible to be installed on every smartphone.

\section{Room for Improvement}

The white color of the background cannot be changed, which can possibly make the eyes tired after prolonged reading.

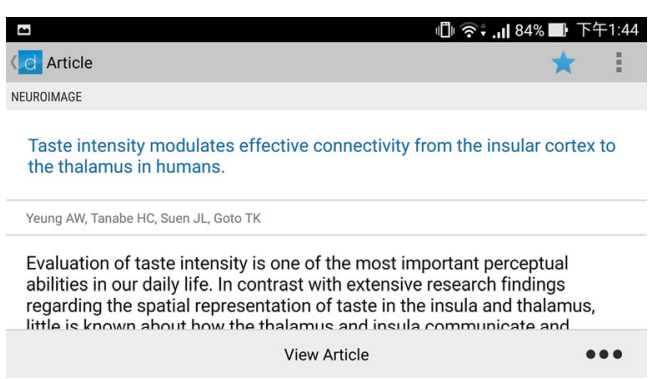

Fig. 6 Landscape mode. The app is compatible with the landscape mode, with same font size and line spacing as the portrait mode

Possibly due to some minor incompatibility with the hardware or software, the app occasionally stops working. Also, users should expect the loading time to be slightly slow with $3 \mathrm{G}$ networks. It is unable to tag or comment on the abstract page of the records.

\section{References}

1. Yeung AWK, Tanabe HC, Suen JL, Goto TK: Taste intensity modulates effective connectivity from the insular cortex to the thalamus in humans. NeuroImage 135:214-222, 2016

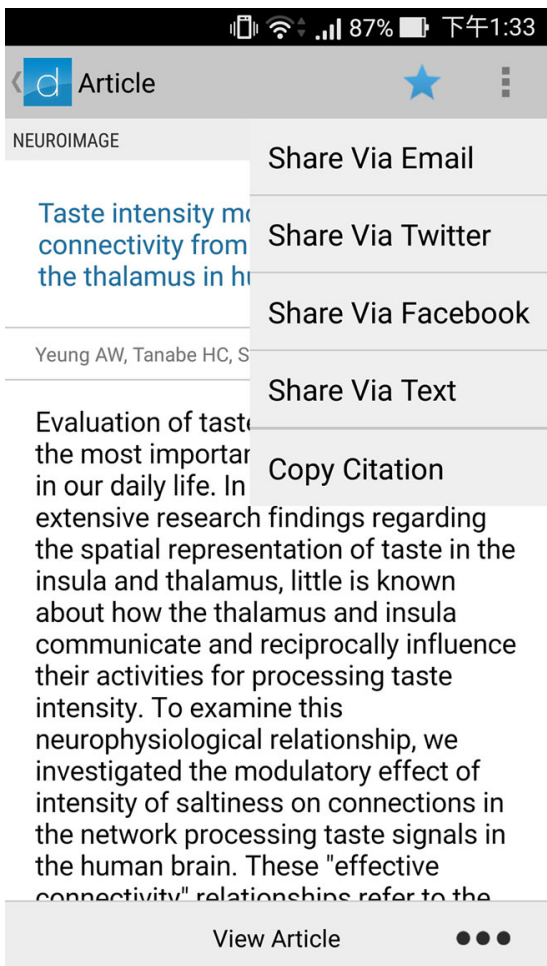

Fig. 5 Portrait mode. An article can be bookmarked by clicking the star icon and can be shared via various channels by clicking the three squares at the upper right corner 